\section{Molecular mechanism of temperature sensing by the circadian clock of Neurospora crassa}

\author{
Axel C.R. Diernfellner, ${ }^{1}$ Tobias Schafmeier, ${ }^{1}$ \\ Martha W. Merrow, ${ }^{2}$ and Michael Brunner ${ }^{1,3}$ \\ ${ }^{1}$ Biochemistry Center, University of Heidelberg (BZH), \\ Heidelberg D-69120, Germany; ${ }^{2}$ Rijksuniversiteit Groningen, \\ Department of Behavioural Biology, 9750 AA Haren, \\ The Netherlands
}

Expression levels and ratios of the long (1) and short (s) isoforms of the Neurospora circadian clock protein FREQUENCY (FRQ) are crucial for temperature compensation of circadian rhythms. We show that the ratio of l-FRQ versus $s-F R Q$ is regulated by thermosensitive splicing of intron 6 of $\mathrm{frq}$, a process removing the translation initiation site of l-FRQ. Thermosensitivity is due to inefficient recognition of nonconsensus splice sites at elevated temperature. The temperature-dependent accumulation of FRQ relative to bulk protein is controlled at the level of translation. The 5'-UTR of frq RNA contains six upstream open reading frames (uORFs) that are in nonconsensus context for translation initiation. Thermosensitive trapping of scanning ribosomes at the uORFs leads to reduced translation of the main ORF and allows adjustment of FRQ levels according to ambient temperature.

Supplemental material is available at http://www.genesdev.org.

Received April 6, 2005; revised version accepted July 4, 2005.

Circadian clocks are cellular oscillators that organize temporal expression of large numbers of genes in many organisms. They are synchronized to the 24-h cycle of earth rotation, and changes in light and ambient temperature are major natural cues (zeitgebers) for entrainment. Without zeitgebers, circadian clocks oscillate with their endogenous free-running periods, which often deviate from $24 \mathrm{~h}$. In fungi, plants, and animals, circadian rhythmicity depends on clock proteins, which interact via interconnected transcriptional/translational feedback loops such that RNA and protein levels of at least one clock gene oscillate (Allada et al. 2001; Reppert and Weaver 2002; Roenneberg and Merrow 2003; Stanewsky 2003; Dunlap and Loros 2004; Gachon et al. 2004). Post-transcriptional mechanisms contribute substantially to the circadian rhythmicity. In particular, assembly, subcellular localization and turnover kinetics of

[Keywords: Circadian clock; temperature compensation; alternative splicing; translational control]

${ }^{3}$ Corresponding author.

E-MAIL michael.brunner@urz.uni-heidelberg.de; FAX 49-6221-544769. Article published online ahead of print. Article and publication date are at http://www.genesdev.org/cgi/doi/10.1101/gad.345905. clock components are regulated by phosphorylation (Stanewsky 2003; Dunlap and Loros 2004; Gachon et al. 2004).

The role of temperature in regulation of circadian rhythmicity is complex and not fully understood. In general, temperature shifts result in the resetting of circadian clocks, i.e., affect the phase of the circadian oscillation. However, while biochemical processes are generally temperature dependent, the free-running periods of circadian rhythms are precise and efficiently temperature compensated with a $\mathrm{Q}_{10}$ close to unity (Izumo et al. 2003; Tsuchiya et al. 2003; Dunlap and Loros 2004). Yet, temperature affects the circadian clock, allowing its adaptation to seasonal variation of day length.

In Drosophila, thermosensitive splicing in the 3 '-UTR of the clock gene period (per) leads to phase advanced accumulation of per RNA on cold days, which is crucial for seasonal adaptation of circadian locomotor activity (Majercak et al. 1999). Thermosensitive splicing is affected by light, the clock, and phospholipase C (Collins et al. 2004; Majercak et al. 2004).

In Neurospora, FREQUENCY (FRQ) is a central component of the circadian clock (Dunlap and Loros 2004). frq RNA is rhythmically expressed and synthesized with a 5'-UTR containing six upstream open reading frames (uORFs) (Liu et al. 1997). A large (1) and a small (s) isoform of FRQ are translated from a common primary transcript. It has been shown that the abundance and ratio of l-FRQ versus s-FRQ are crucial for robust free-running rhythmicity, as well as for temperature compensation of the circadian clock (Garceau et al. 1997; Liu et al. 1997). l-FRQ versus s-FRQ is expressed in a temperature-dependent fashion. Expression of 1-FRQ relative to bulk protein increases with increasing temperature, while expression of s-FRQ is essentially temperature independent. The underlying molecular mechanisms by which Neurospora senses temperature to adjust levels and ratio of 1-FRQ versus s-FRQ are not known. It has been proposed that ribosomes scan more efficiently through the AUG codon of l-FRQ at low temperature and thus favor initiation at the downstream in-frame AUG of s-FRQ (Liu et al. 1997). No mechanism has been proposed for the steep temperature dependence of 1-FRQ abundance relative to bulk protein.

Here we have analyzed the molecular mechanisms underlying temperature-dependent levels and ratios of 1FRQ versus s-FRQ. We show that the ratio of $1-F R Q$ versus s-FRQ is regulated by thermosensitive splicing of intron 6 (I-6) of frq RNA. Thermosensitivity is a direct consequence of inefficient recognition of nonconsensus splice sites by the splicing machinery at elevated temperature. Preferential splicing of I-6 at low temperature results in excision of the translation initiation site of l-FRQ, such that the I-6 spliced messages encode only s-FRQ. Interconnected with thermosensitive splicing is a temperature-dependent translational control mechanism that is crucial for the increase in FRQ relative to bulk protein with increasing temperature. The Kozak sequences of the uORFs in the $5^{\prime}$-UTR of frq are in nonconsensus context for translation initiation. As a consequence, the uORFs are more efficiently translated at low temperature, leading to a reduction of translation initiation at the downstream main ORF encoding 1- and s- 
FRQ. The thermosensitive trapping of scanning ribosome at of the uORFs allows adjustment of FRQ levels according to ambient temperature.

\section{Results and Discussion}

Transcription initiation sites of the frq promoter were determined by cap-specific 5'-RNA ligase-mediated rapid amplification of cDNA ends (5'RLM-RACE). A number of different sites were identified $\sim 1.5 \mathrm{~kb}$ upstream of the frq ORF, the major one at -1519 (Fig. 1A). This site is $156 \mathrm{bp}$ downstream of the proximal lightresponsive element of the frq promoter (Froehlich et al. 2002). A putative CCAAT box (Smail and Kadonga 2003) is located at -201 . No obvious TATA box was identified.

Since the $5^{\prime}$-UTR of the primary fr $q$ transcript contains a number of putative splice sites, cDNA was analyzed by polymerase chain reaction (PCR). Six different introns, giving rise to seven splice-isoforms of frq RNA, were identified (Fig. 1B; Supplementary Table 1). Interestingly, splicing of I-6 removes the translation initia-

A

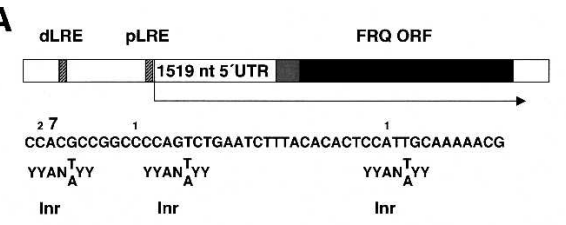

B

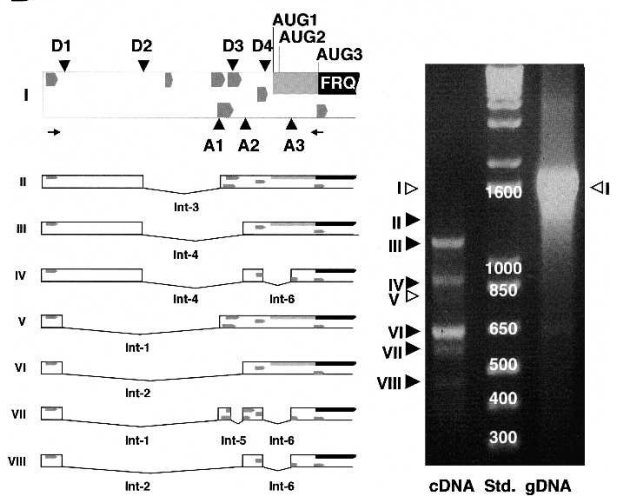

Figure 1. The 5'-UTR of frq RNA is spliced in a complex fashion. $(A$, top $)$ The frq gene is schematically outlined. Distal light-responsive elements (dLRE) and proximal light-responsive elements (pLRE) are indicated by hatched boxes (Froehlich et al. 2002). The major transcription initiation site is indicated by an arrow. The black and the gray + black areas correspond to s-FRQ and l-FRQ, respectively. (Bottom) Major and minor transcription initiation sites. Eleven independent clones of cap-specific RLM-RACE products were sequenced. Numbers above the sequence indicate the 5 '-ends of these clones. The major initiation site (seven clones) corresponds to position -1519. Initiator consensus sequences (Inr) for transcription (Smail and Kadonga 2003) are indicated below the sequence. (Y indicates $\mathrm{C}$ or $\mathrm{T} ; \mathrm{N}$ indicates any nucleotide). (B, top) Schematic outline of the $5^{\prime}$-UTR of frq. Splice donor (D) and acceptor (A) sites and translation initiation sites for l-FRQ (AUG1) and s-FRQ (AUG3) are shown. AUG2 is not used for initiation (Liu et al. 1997). Dark-gray arrows indicate UORF1 to uORF6 in the 5'-UTR and ORF7 overlapping the AUG of s-FRQ. The arrows below the scheme indicate oligonucleotide primers for amplification of cDNA. The outlined unspliced (I) and spliced (II-VIII) frq RNA species (left panel) were amplified by reverse transcription and PCR (right panel) and identified by sequencing (black arrowheads) or by diagnostic PCR (white arrowheads). RNA was prepared from cells grown at $25^{\circ} \mathrm{C}$ in LL. (Std) DNA size standard; (gDNA) genomic DNA.
A
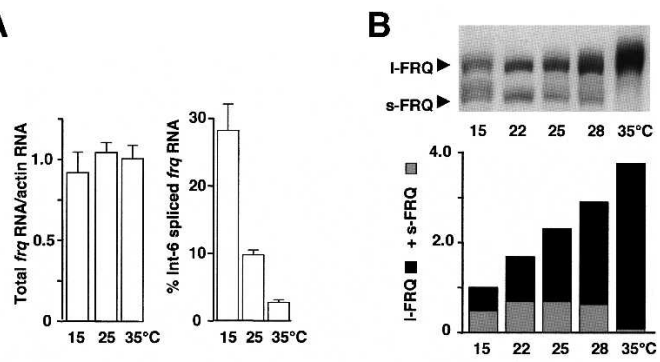

C

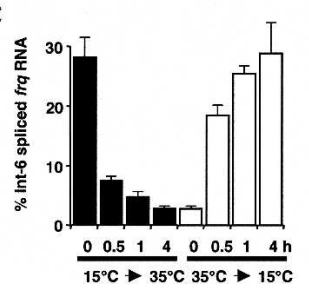

D

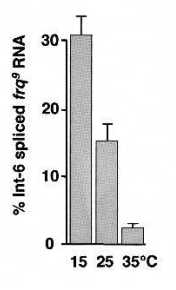

Figure 2. I-6 is spliced in a temperature-dependent fashion. $(A)$ Neurospora was grown at the indicated temperatures in LL, and RNA was prepared and quantitfied by RT-PCR (see Materials and Methods). (Left) Total frq RNA was determined. frq RNA/actin RNA at $35^{\circ} \mathrm{C}$ was set equal to 1 . (Right) The fraction (\%) of frq RNA spliced at I-6 (spliced/total frq RNA) was determined. (B) Protein extracts prepared from Neurospora that was grown at the indicated temperatures in LL were treated with alkaline phosphatase. Samples were analyzed by immunoblotting with $\alpha \mathrm{FRQ}$, recognizing l-FRQ and s-FRQ (top) and quantified by densitometry (bottom). l-FRQ (black bars) and s-FRQ (gray bars) are shown. (C) Cultures were shifted from $15^{\circ} \mathrm{C}$ to $35^{\circ} \mathrm{C}$ (dark bars) or from $35^{\circ} \mathrm{C}$ to $15^{\circ} \mathrm{C}$ (white bars). The fraction of spliced I-6 was determined. $(D)$ Thermosensitive splicing of I-6 is independent of functional FRQ. fr $^{9}$ was grown in LL at the indicated temperatures. RNA was prepared, and splicing of I-6 was determined.

tion site of 1-FRQ. The resulting fr $q$ RNA species encode s-FRQ specifically. The Lariat sequence of I-6 deviates considerably from consensus (Supplementary Table 1), and only a fraction of $f r q$ RNA was spliced at I- 6 . This suggests that 1-FRQ and s-FRQ are translated from alternatively spliced messages. Since the ratio of l-FRQ versus s-FRQ is temperature dependent (Garceau et al. 1997), Neurospora was grown at different temperatures in constant light (LL) and RNA was analyzed. Total levels of $f r q$ RNA were constant between $15^{\circ} \mathrm{C}$ and $35^{\circ} \mathrm{C}$ (Fig. 2A), in agreement with measurements of frq RNA at $21^{\circ} \mathrm{C}$ and $28^{\circ} \mathrm{C}$ (Liu et al. 1998). Splicing of I- 1 through I-5 was temperature independent (data not shown). However, splicing of I-6 was temperature sensitive with a $Q_{10}$ of 3 (Fig. 2A,D). Corresponding results were obtained when Neurospora was grown in darkness for $24 \mathrm{~h}$ (data not shown), demonstrating that thermosensitive splicing was not light dependent.

Splicing of I-6 correlates with expression of 1-FRQ versus s-FRQ. Expression of 1-FRQ increased with increasing temperature, while s-FRQ was expressed at low levels between $15^{\circ} \mathrm{C}$ and $35^{\circ} \mathrm{C}$ (Fig. 2B). Data are essentially in agreement with earlier reports for dark-grown Neurospora (Garceau et al. 1997; Liu et al. 1997). Thus, the fraction of $f_{r} q$ RNA spliced at I-6 correlates with relative expression levels of l-FRQ versus s-FRQ.

We investigated the kinetics of I-6 splicing upon temperature shifts. When cultures were shifted from $15^{\circ} \mathrm{C}$ to $35^{\circ} \mathrm{C}$, the fraction of spliced I-6 decreased within $30 \mathrm{~min}$ 
from $28 \%$ to $7 \%$ and reached a value $<3 \%$ after $4 \mathrm{~h}$ (Fig. 2C). Correspondingly, upon shift from $35^{\circ} \mathrm{C}$ to $15^{\circ} \mathrm{C}$, the fraction of spliced I- 6 increased rapidly from $\sim 3 \%$ to $18 \%$ after $30 \mathrm{~min}$ and to $30 \%$ after $4 \mathrm{~h}$ (Fig. 2C). The fast adaptation of $f_{r} q$ RNA splicing to ambient temperature suggests that the process may not require de novo protein synthesis.

The mutant $f_{r} q^{9}$ allele expresses a full-size RNA that encodes (due to a premature stop) a nonfunctional, Cterminally truncated protein (Aronson et al. 1994). I-6 of $f r q^{9}$ RNA was spliced in a temperature-dependent fashion (Fig. 2D), demonstrating that FRQ is not required for this function.

Next, splice sites of I-6 were optimized toward consensus $\left(I-6^{o p t}\right)$ and mutagenized $\left(I-6^{m u t}\right)$ to nonsplice sites (Supplementary Table 1) by site-directed mutagenesis, and frq RNA and FRQ were analyzed. I-6 ${ }^{\text {opt }}$ was efficiently spliced in a temperature-independent fashion (Fig. 3A). s-FRQ was synthesized at all temperatures, and only trace amounts of l-FRQ were detected (Fig. 3B,C). In contrast, $I-6^{\text {mut }}$ was not spliced (Fig. 3A). 1-FRQ was synthesized, and little s-FRQ was detected (Fig. 3B,C). Thus, in $f_{r} q^{+}$, s-FRQ is primarily synthesized from $f_{r q}$ RNA species spliced at I-6.

How does splicing of I-6 affect temperature compensation of the circadian clock? The free-running period of Neurospora can be determined by analyzing the conidiation rhythm (banding) on race tubes. Banding can be observed most clearly between $22^{\circ} \mathrm{C}$ and $28^{\circ} \mathrm{C}$. The freerunning period of wild type decreases only slightly between $22^{\circ} \mathrm{C}$ and $28^{\circ} \mathrm{C}$ (Fig. 3D; Supplementary Fig. 1), demonstrating that the clock is well temperature compensated. The period of the $I-6^{\text {mut }}$ strain decreased steeper compared with wild type, while the period of the $I-6^{\text {opt }}$ strain increased. The data suggest that l-FRQ tends to shorten the period while s-FRQ causes period lengthening.

The data suggest that the molecular basis for the temperature-dependent splicing of I-6 is provided by the interaction of the splicing machinery with nonconsensus splice sites, which are more efficiently recognized at low temperature.

To test this hypothesis, we inserted into the frq ORF I- 8 of the period gene (dmpi8), which is spliced in a thermosensitive fashion in Drosophila (Majercak et al. 1999). The chimeric frq-dmpi8 allele was expressed at different temperatures, and splicing was analyzed by qualitative RT-PCR (Fig. 3E). Like I-6, dmpi8 was also spliced in a thermosensitive fashion.

It should be pointed out that the ratio of 1-FRQ versus s-FRQ correlates with the fraction of spliced I-6, while the increase of overall FRQ levels with increasing temperature is independent of I-6. Temperature-dependent expression of FRQ does not correlate with levels of frq RNA, which are temperature independent (Fig. 2, cf. A and B). Apparently, FRQ levels are regulated in a posttranscriptional fashion.

What is the molecular basis for the temperature dependence of FRQ accumulation? We constructed a strain where the long $5^{\prime}$-UTR of the frq gene was deleted (Fig. 4A). In this strain, FRQ levels increased only by a factor of 1.4 between $15^{\circ} \mathrm{C}$ and $35^{\circ} \mathrm{C}$, suggesting that the $5^{\prime}$ UTR affects the temperature-dependent expression of FRQ. frq RNA levels increased in a similar fashion (Supplementary Fig. 2A). FRQ levels were significantly lower in $f_{r q} \Delta \mathrm{UTR}$ than in $\mathrm{fr}^{+}$, while RNA levels were
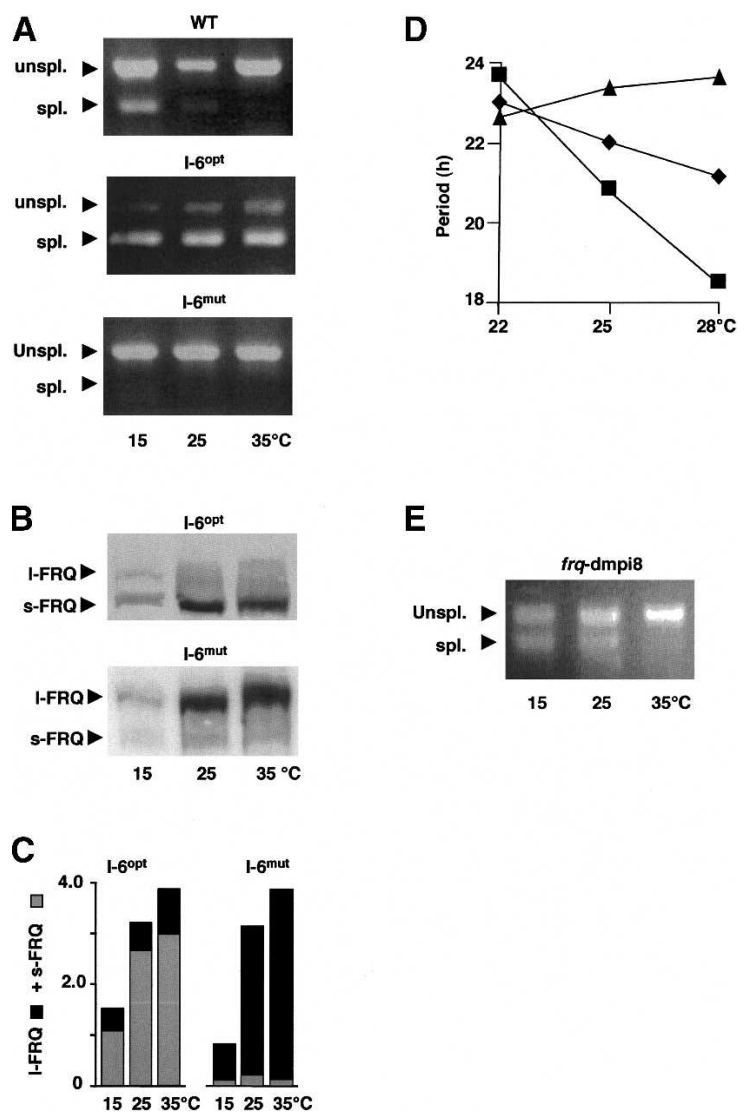

Figure 3. Splice-site mutations affecting thermosensitive splicing of I- 6 . The splice sites of I- 6 were altered by site-directed mutagenesis toward consensus (I- $\left.6^{\text {opt }}\right)$ and to nonsplice sites (I- $\left.6^{\text {mut }}\right)$ as indicated in Supplementary Table 1. (A) Reverse transcription and qualitative PCR analysis showing that I- $6^{\text {opt }}$ is efficiently spliced at all temperatures while I- $6^{\text {mut }}$ is not spliced. $(B)$ l-FRQ is the major protein product synthesized from the I- $6^{\text {mut }}$ allele and s-FRQ from I-6 ${ }^{\text {opt }} .(C)$ Quantification of Western blots shown in B. (Black bars) l-FRQ; (gray bars) s-FRQ. (D) The free-running period of I-6 ${ }^{\text {opt }}$ (triangles), I-6 ${ }^{\text {mut }}$ (squares), and wild-type (diamonds) control strains was determined on race tubes at the indicated temperatures. $(E)$ I-8 of the Drosophila per gene (dmpi8) was inserted into the unique AflII site of the fr $q$ gene. The fr $q$-dmpi8 allele was expressed in a fr $q^{10}$ background. Reverse transcription and qualitative PCR analysis showing that dmpi8 is spliced in a thermosensitive fashion in Neurospora.

similar. This may be due to a reduced efficiency of translation of frq $\Delta \mathrm{UTR}$, which is often observed when the translation initiation site is close to the $5^{\prime}$-end of the RNA (Hinnebusch and Natarajan 2002; Vilela and McCarthy 2003).

We noticed that the uORFs in the 5'-UTR of $f r q$ are in nonconsensus context for translation initiation (Supplementary Table 2; Bruchez et al. 1993), suggesting that they are inefficiently translated. If translation initiation at the uORFs were more efficient at low temperature, trapping of ribosomes (Gaba et al. 2001; Hinnebusch and Natarajan 2002; Vilela and McCarthy 2003; Gebauer and Hentze 2004) could provide a mechanism for the temperature-dependent expression of the main ORF.

A major species of $f r q$ RNA is spliced at I-2 and contains uORF1 and uORF6 (Fig. 1). We changed the AUG codons of uORF1 and uORF6 to GUG $\left(f_{r q} 1 / 6^{\text {mut }}\right)$ to prevent translation. Expression of FRQ was less tempera- 
A
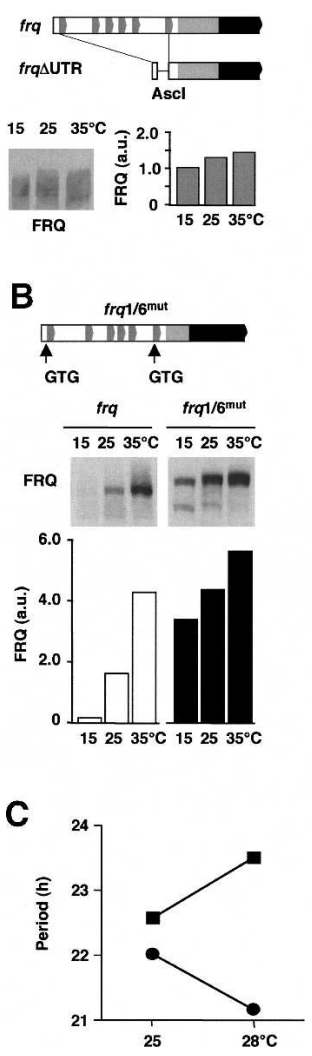

Figure 4. uORFs restrict translation of FRQ. (A) In frq $\Delta$ UTR residues, 50-1477 of the 5'-UTR are deleted and an AscI site is introduced. Western blot and densitometric quantification showing temperature-dependent expression of FRQ-encoded frq $\Delta$ UTR. (B) Construction $\mathrm{fr} q 1 / 6^{\mathrm{mut}}$ : Start codons of uORF1 and uORF6 were altered by site-directed mutagenesis to GTG. Temperature-dependent expression of FRQ encoded by $f r q$ and $f r q 1 / 6^{\text {mut }}$ is shown. $(C)$ The free-running period of $f r q 1 / 6^{\text {mut }}$ (squares) and wild-type (circles) control $(f r q)$ was determined on race tubes at $25^{\circ} \mathrm{C}$ and $28^{\circ} \mathrm{C}$. (D) Construction of $\Delta \mathrm{I}-2$ fr $q 1 / 6^{\mathrm{AUG}}$ and $\Delta \mathrm{I}-2$ fr $q 1 / 6^{\mathrm{mut}}$ : cDNA derived from I-2 spliced $f r q$ RNA was used as template for PCR reactions. Fragments including the translation initiation site of uORF1 and uORF6 were amplified by PCR and inserted into the AscI site of frq $\Delta \mathrm{UTR}$. Start codons of uORF1 and uORF6 were either left unchanged $(\Delta \mathrm{I}-2$ frq $\left.1 / 6^{\mathrm{AUG}}\right)$ or changed to TTG and ACG $\left(\Delta \mathrm{I}-2\right.$ frq $\left.1 / 6^{\mathrm{mut}}\right)$. In the constructs shown, the $\mathrm{C}$ terminus of uORF6 (. . HKLLERE) was

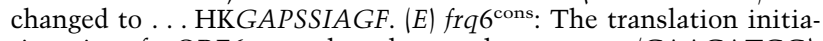
tion site of uORF6 was altered toward consensus (CAACATGG), and expression of FRQ was measured at the indicated temperatures. The ratios of FRQ expressed by $f_{r} q 6^{\text {cons }}$ vs. FRQ expressed by a corresponding wild-type frq allele are shown. $(F)$ Conidiation of the strain $f r q 6^{\text {cons }}$. Race tubes were inoculated in LL and incubated at $25^{\circ} \mathrm{C}$ in DD. After $24 \mathrm{~h}$, they were transferred to the indicated temperatures.

ture dependent in $f_{r q} 1 / 6^{\text {mut }}$ than in a frq control strain (Fig. 4B). At $15^{\circ} \mathrm{C}$, FRQ levels were significantly higher in $f r q 1 / 6^{\text {mut }}$, while the levels were similar in both strains at $35^{\circ} \mathrm{C}$. fr $q 1 / 6^{\mathrm{mut}}$ RNA levels were independent of temperature (Supplementary Fig. 2B). This indicates that the uORFs reduce expression of FRQ preferentially at low temperature.

The frq $1 / 6^{\text {mut }}$ strain showed no clear conidiation rhythm at $\leq 22^{\circ} \mathrm{C}$ (data not shown). At $25^{\circ} \mathrm{C}$ and $28^{\circ} \mathrm{C}$, the period was $\sim 0.5 \mathrm{~h}$ and $2 \mathrm{~h}$ longer compared with a frq control strain (Fig. 4C).

We then constructed two frq alleles where a region corresponding to I-2 was deleted (Fig. 4D). In one, the AUG codons of uORF1 and uOFR6 were left unchanged $\left(\Delta \mathrm{I}-2 \mathrm{fr} q 1 / 6^{\mathrm{AUG}}\right)$, and in the other, they were mutated $\left(\Delta \mathrm{I}-2 \mathrm{frq} 1 / 6^{\mathrm{mut}}\right)$. In addition, the splice donor of I-6, which is located in uORF6, was deleted (Materials and Methods), resulting in an altered $\mathrm{C}$ terminus of uORF6. Expression of FRQ encoded by $\Delta \mathrm{I}-2$ fr $q 1 / 6^{\mathrm{AUG}}$ exhibited similar temperature dependence as $\mathrm{fr}^{+}$(Fig. 4D), whereas FRQ levels were significantly higher in $\Delta \mathrm{I}-2$ frq $1 / 6^{\text {mut }}$ and essentially independent of temperature.

Finally, we altered the translation initiation site of UORF6 toward consensus by site-directed mutagenesis. FRQ levels in the $\mathrm{fr}_{\mathrm{r}} \mathrm{6}^{\mathrm{cons}}$ strain were substantially lower and less temperature dependent than in a corresponding control strain (Fig. 4E). The conidiation pattern of frq $6^{\text {cons }}$ was obscured at all three temperatures (Fig. 4F), presumably due to the low amplitude of FRQ oscillation.

Together, the data demonstrate that uORFs in the $5^{\prime}$ UTR restrict translation of the downstream frq ORF, in particular at low temperature. The temperature-dependent expression of FRQ is regulated by translation of uORFs, in particular uORF1 and uORF6, rather than by putative secondary structure elements in the $5^{\prime}$-UTR (Gebauer and Hentze 2004).

This suggests a model in which temperature dependence of expression levels and ratios of 1-FRQ versus sFRQ is regulated by two distinct thermosensitive processes. The ratio of 1-FRQ versus s-FRQ is regulated by thermosensitive splicing of I-6, removing the translation initiation site of 1-FRQ. Temperature-dependent accumulation of FRQ $(1+s)$ is regulated by thermosensitive translation initiation at uORFs in the $5^{\prime}$-UTR. In both cases, temperature assessment appears to be a direct consequence of low-affinity interactions of general machinery for splicing and translation with nonconsensus lariat and Kozak sequences, respectively.

When the splice sites of I-6 were mutated toward consensus, I-6 was efficiently spliced in a temperature-independent fashion. Thus, the splice sites are cis-acting elements conferring a temperature-sensitive phenotype in regards to removal of I- 6 . However, while reduced splicing of I-6 at high temperature can be overcome by mutation $\left(I-6^{\text {opt }}\right)$, there may be another sequence element or factor that contributes to the inhibition of I-6 splicing at high temperatures.

Temperature-dependent splicing of I-6 is independent of FRQ and thus probably independent of the clock. Thermosensitive splicing was reported for I-8 (dmpi8) in the 3'-UTR of the circadian clock gene period (per) of Drosophila (Majercak et al. 1999). The temperature-dependent mean splicing efficiency of dmpi8 appears to be also clock independent (Majercak et al. 2004). Splice sites of I-6 (lariat) and dmpi8 (acceptor) deviate from consensus, and both introns are spliced in a temperaturedependent fashion in Neurospora. This suggests that the molecular basis of thermosensitive splicing may be similar in frq and per. Thermosensitive splicing of per is modulated by the circadian clock and by phospholipase C via unknown pathways (Collins et al. 2004; Majercak et al. 2004). Preliminary results suggest that splicing of fr $q$ is also modulated by the clock (data not shown).

In contrast to splicing of I-6, splicing of dmpi8 does not lead to expression of different PER isoforms. Rather, the splicing event per se leads to more efficient maturation and accumulation of per RNA compared with splice-deficient and intron-less mutants (Majercak et al. 1999). 
Preferential splicing of dmpi8 at low temperature leads to more efficient accumulation of per RNA. As a consequence, critical PER levels accumulate earlier, leading to a phase advanced locomotor activity of Drosophila at seasonally cold days. Whether and how thermosensitive splicing of frq I-6 affects seasonal adaptation of the Neurospora clock remains to be analyzed.

Recently, it has been reported that FRQ is in a complex with FRH, a homolog of an RNA helicase associated with the exosome (Cheng et al. 2005). The exosome is a multisubunit complex involved in 3 '-trimming of different RNA species, including mRNA /van Hoof et al. 2000). However, splicing of I-6 is temperature dependent in frq-deficient strains $\left(f r q^{9}\right)$, demonstrating that the FRQ/FRH complex is not required for thermosensitive splicing of $f r q$ RNA.

While the ratio of 1-FRQ versus s-FRQ is regulated via thermosensitive splicing, the temperature-dependent accumulation of both isoforms of FRQ is regulated on translational level. uORFs in the 5'-UTR of frq affect expression of the main ORF encoding 1- and s-FRQ in a temperature-dependent manner. When the AUGs of uORF1 and UORF6 are eliminated, temperature-dependent accumulation of FRQ is less pronounced; FRQ levels are elevated at low temperature but similar to wild type at high temperature. Thus, uORFs reduce FRQ expression at low temperature but have little effect at high temperature. The translation initiation sites of the uORFs deviate from consensus Kozak sequences. We suggest that the translation machinery may more efficiently initiate at the nonconsesus Kozak sequences at low temperature, while the uORFs are only inefficiently translated at elevated temperatures.

$\mathrm{uORFs}$ were reported to regulate translation of downstream located genes in a number of different ways. In the arg-2 gene of Neurospora and in the CPA1 gene of Saccharomyces cerevisiae, uORFs encode specific arginine-attenuator peptides that cause stalling of ribosomes (Gaba et al. 2001; Vilela and McCarthy 2003; Gebauer and Hentze 2004). Although uORFs are also present in frq genes of filamentous fungi related to Neurospora, the encoded peptides are not conserved (data not shown). It seems therefore unlikely that the UORF peptides cause ribosome stalling as in arg-2. The length of a uORF and the sequence context in the vicinity of the stop codon affect whether translating ribosomes terminate and fall off or resume scanning downstream of the uORF. The uORFs in $f r q$ are quite short, so ribosomes could, in principle, resume the scanning process after uORF translation. However, the sequences surrounding the stop codons of uORF1 and uORF6 are GC-rich, which is thought to favor dissociation and release of terminating ribosomes as reported for uORF4 in the GCN4 gene of $S$. cerevisiae (Gaba et al. 2001; Hinnebusch and Natarajan 2002; Vilela and McCarthy 2003; Gebauer and Hentze 2004). Yet, the precise mechanism by which the uORFs regulate thermosensitive translation of the downstream frq ORF remains to be investigated.

When ratio of 1 -FRQ versus s-FRQ was manipulated via altered splicing, temperature compensation of the period length was affected. Although we cannot attribute distinct molecular functions to the FRQ isoforms, s-FRQ seems to cause period shortening and l-FRQ period lengthening (Fig. 3D). When uORF1 and uORF6 were mutagenized, the levels of FRQ increased. The free-running rhythmicity of the $f r q 1 / 6^{\text {mut }}$ strain was lost in the

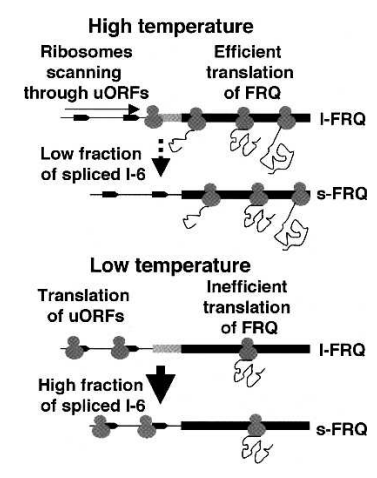

Figure 5. Model for temperature sensing by the circadian clock via nonconsensus splicing of $f r q$ RNA and nonconsensus translation initiation at uORFs. For details, see Discussion.

low temperature range, and the period was lengthened at $28^{\circ} \mathrm{C}$. This suggests that elevated FRQ levels maintain a prolonged repression of the frq locus and thus cause period lengthening. Reduction of FRQ expression by optimizing translation of uORF6 resulted in an obscured conidiation pattern, suggesting that the amplitude of FRQ oscillation is crucial for a robust overt rhythmicity.

Why is the expression of FRQ then regulated in such a complex fashion? In Drosophila, rhythmicity of the clock is not affected when splicing of per is challenged. Rather, thermosensitive splicing of per regulates the adaptation of locomotor activity to seasonally warm and long versus cold and short days. Accordingly, splicing and translational control of $f r q$ may contribute to finetuning of clock functions in Neurospora, modulating, in particular, adaptation of the circadian clock to seasonal variations of photoperiod and temperature (Tan et al. 2004a,b).

How may levels and ratios of 1-FRQ versus s-FRQ contribute to temperature compensation of period length? To reliably measure time on the molecular level, it might be necessary that FRQ-dependent biochemical processes proceed at a constant net rate at all temperatures. Since protein-protein interactions are generally temperature dependent, a decrease in complex stability at elevated temperature must be compensated. The increased expression of FRQ compared with bulk protein could account for such compensation. In addition, the preferential expression of 1-FRQ over s-FRQ at high temperature could reflect accumulation of a FRQ isoform that is capable of forming more stable interactions. This is supported by the observation that Neurospora mutants that express low levels of s-FRQ but no l-FRQ are arrhythmic on race tubes at elevated temperatures but become rhythmic when s-FRQ is expressed at elevated levels (Liu et al. 1997).

We suggest a molecular mechanism for temperaturesensing resulting in adjustment of expression levels and ratios of 1-FRQ versus s-FRQ (Fig. 5): In the high-temperature range, ribosomes are scanning through the nonconsensus AUGs of the uORFs in the $5^{\prime}$-UTR of $f r q$ and initiate translation at the main ORF, leading to efficient expression of FRQ. At low temperatures, scanning ribosomes initiate translation more efficiently at the uORFs, leading to a reduced translation of the downstream frq ORF. Accordingly, overall expression of FRQ $(1+s)$ decreases with decreasing temperature. Superimposed on this translational control is the thermosensitive splicing 
of the nonconsensus I-6, which is more efficiently spliced at low temperatures. Since I-6 spliced frq RNA encodes specifically s-FRQ, the ratio of l-FRQ versus sFRQ is decreasing with decreasing temperature. Thus, the strategy used to measure temperature on the molecular levels appears to be the use of nonconsensus signals for splicing and translation initiation. Since these signals form weaker interactions with the splicing machinery and with ribosomes, respectively, than corresponding consensus signals, they are more efficiently recognized at low temperatures and thus provide a molecular temperature sensor.

\section{Materials and methods}

Strains

Recombinant frq alleles were generated by site-directed mutagenesis (Quickchange XL Site-Directed Mutagenesis Kit, Stratagene) of the ClaI fragment carrying the frq locus (McClung et al. 1989); inserted into the his-3 locus of $f_{r q}{ }^{10}$, his-3, bd; and strains were grown in LL as described (Görl et al. 2001).

Donor (D4), Lariat, and Acceptor (A3) sequences of I-6 were either changed toward consensus or mutagenized to nonsplice sites using the oligonucleotides shown in the Supplemental Material.

Construction of frq $\Delta U T R$, mutagenesis of uORFs and construction of frq-dmpi8 is described in the Supplemental Material.

\section{RNA analysis}

cDNA was synthesized from $4 \mu \mathrm{g}$ DNase-treated total RNA using the SuperScriptII RT-system (Invitrogen) and gene-specific reverse primers. fr $q$ cDNA and actin cDNA was detected by quantitative real-time PCR (ABI-Prism 7000, Applied Biosystems) using specific primers and TaqMan probes (Görl et al. 2001). Primers and probes for quantification of I-6 spliced and unspliced RNA are shown in Supplemental Material. At least triplicate reactions $(25 \mu \mathrm{L})$ containing cDNA equivalent to $0.1 \mu \mathrm{g}$ RNA were analyzed.

\section{Protein analysis}

Protein extracts were prepared as described (Görl et al. 2001). For dephosphorylation extracts, $5 \mathrm{mg} / \mathrm{mL}$ were incubated with alkaline phosphatase $(3000 \mathrm{U} / \mathrm{mL})$ for $1 \mathrm{~h}$ at $37^{\circ} \mathrm{C}$. Protein was separated by SDS-PAGE $(7.5 \%)$, and Western blotting was performed (Görl et al. 2001). Affinity-purified polyclonal antibodies directed against the C-terminal 167-amino-acid residues of FRQ were used together with peroxidase-coupled secondary antibody. Enhanced chemiluminescence signals were detected with Xray films and quantified by densitometry.

\section{Acknowledgments}

This work was supported by grants from Deutsche-Forschungsgemeinschaft (BR 1375-1 and SFB 638) and by Fonds der Chemischen Industrie.

\section{References}

Allada, R., Emery, P., Takahashi, J.S., and Rosbash, M. 2001. Stopping time: The genetics of fly and mouse circadian clocks. Annu. Rev. Neurosci. 24: 1091-1119.

Aronson, B.D., Johnson, K.A., and Dunlap, J.C. 1994. Circadian clock locus frequency: Protein encoded by a single open reading frame defines period length and temperature compensation. Proc. Natl. Acad. Sci. 91: 7683-7687.

Bruchez, J.J.P., Eberle, J., and Russo, V.E.A. 1993. Regulatory sequences involved in the translation of Neurospora crassa mRNA: Kozak sequences and stop codons. Fungal Genet. Newslett. 40: 85-88.

Cheng, P., He, Q., He, Q., Wang, L., and Liu, Y. 2005. Regulation of the Neurospora circadian clock by an RNA helicase. Genes \& Dev. 19: 234-241.

Collins, B.H., Rosato, E., and Kyriacou, C.P. 2004. Seasonal behavior in Drosophila melanogaster requires the photoreceptors, the circadian clock, and phospholipase C. Proc. Nat1. Acad. Sci. 101: 1945-1950.

Dunlap J.C. and Loros, J.J. 2004. The Neurospora circadian system. I. Biol. Rhythms 19: 414-424.
Froehlich, A.C., Liu, Y., Loros, J.J., and Dunlap J.C. 2002. White Collar-1, a circadian blue light photoreceptor, binding to the frequency promoter. Science 297: 815-819.

Gaba, A., Wang, Z., Krishnamoorthy, T., Hinnebusch, A.G., and Sachs, M.S. 2001. Physical evidence for distinct mechanisms of translational control by upstream open reading frames. EMBO J. 20: 64536463.

Gachon, F., Nagoshi, E., Brown, S.A., Ripperger, J., and Schibler, U. 2004. The mammalian circadian timing system: From gene expression to physiology. Chromosoma 113: 103-112.

Garceau, N.Y., Liu, Y., Loros, J.J., and Dunlap J.C. 1997. Alternative initiation of translation and time-specific phosphorylation yield multiple forms of the essential clock protein frequency. Cell 89: 469-476.

Gebauer, F. and Hentze, M.W. 2004. Molecular mechanisms of translational control. Nat. Rev. Mol. Cell. Biol. 5: 827-834.

Görl, M., Merrow, M., Huttner, B., Johnson, J., Roenneberg, T., and Brunner, M. 2001. A PEST-like element in FREQUENCY determines the length of the circadian period in Neurospora crassa. EMBO J. 20: 7074-7084.

Hinnebusch, A.G. and Natarajan, K. 2002. Gcn4p, a master regulator of gene expression, is controlled at multiple levels by diverse signals of starvation and stress. Eukaryot. Cell 1: 22-32.

Izumo, M., Johnson, C.H., and Yamazaki, S. 2003. Circadian gene expression in mammalian fibroblasts revealed by real-time luminescence reporting: Temperature compensation and dampening. Proc. Natl. Acad. Sci. 100: 16089-16094.

Liu, Y., Garceau, N.Y., Loros, J.J., and Dunlap J.C. 1997. Thermally regulated translational control of FRQ mediates aspects of temperature responses in the Neurospora circadian clock. Cell 89: 477-486.

Liu, Y., Merrow, M., Loros, J.J., and Dunlap, J. 1998. How temperature resets a circadian oscillator. Science 281: 825-829.

Majercak, J., Sidote, D., Hardin, P.E., and Edery, I. 1999. How a circadian clock adapts to seasonal decreases in temperature and day length. Neuron 24: 219-230.

Majercak, J., Chen, W.F., and Edery, I. 2004. Splicing of the period gene 3 '-terminal intron is regulated by light, circadian clock factors, and phospholipase C. Mol. Cell. Biol. 24: 3359-3372.

McClung, C.R., Fox, B.A., and Dunlap, J.C. 1989. The Neurospora clock gene frequency shares a sequence element with the Drosophila clock gene period. Nature 339: 558-562.

Reppert, S.M. and Weaver, D.R. 2002. Coordination of circadian timing in mammals. Nature 418: 935-941.

Roenneberg, T. and Merrow, M. 2003. The network of time: Understanding the molecular circadian system. Curr. Biol. 13: R198-R207.

Smail, S.T. and Kadonga, J.T. 2003. The RNA polymerase II core promoter. Annu. Rev. Biochem. 72: 449-479.

Stanewsky, R. 2003. Genetic analysis of the circadian system in Drosophila melanogaster and mammals. J. Neurobiol. 54: 111-147.

Tan, Y., Dragovic, Z., Roenneberg, T., and Merrow, M. 2004a. Entrainment dissociates transcription and translation of a circadian clock gene in Neurospora. Curr. Biol. 145: 433-438.

Tan, Y., Merrow, M., and Roenneberg, T. 2004b. Photoperiodism in Neurospora crassa. J. Biol. Rhythms 19: 135-143.

Tsuchiya, Y., Akashi, M., and Nishida, E. 2003. Temperature compensation and temperature resetting of circadian rhythms in mammalian cultured fibroblasts. Genes Cells 8: 713-720.

van Hoof, A., Lennertz, P., and Parker, R. 2000. Yeast exosome mutants accumulate 3 '-extended polyadenylated forms of U4 small nuclear RNA and small nucleolar RNAs. Mol. Cell. Biol. 20:441-452.

Vilela, C. and McCarthy, J.E.G. 2003. Regulation of fungal gene expression via short open reading frames in the mRNA 5'-untranslated region. Mol. Microbiol. 49: $859-867$. 


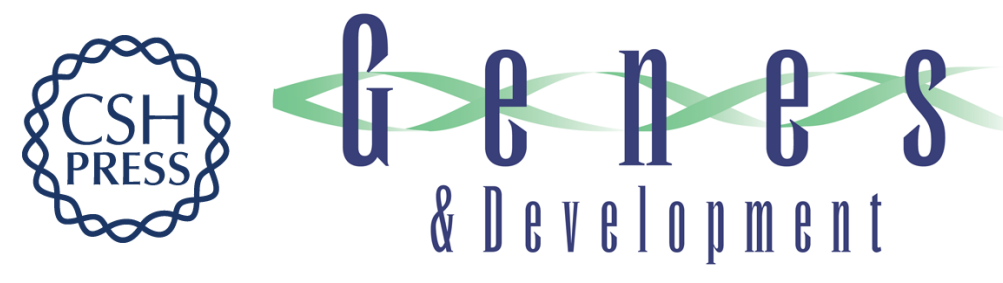

\section{Molecular mechanism of temperature sensing by the circadian clock of Neurospora crassa}

Axel C.R. Diernfellner, Tobias Schafmeier, Martha W. Merrow, et al.

Genes Dev. 2005, 19:

Access the most recent version at doi:10.1101/gad.345905

\section{Supplemental http://genesdev.cshlp.org/content/suppl/2005/08/18/gad.345905.DC1 Material}

References This article cites 27 articles, 11 of which can be accessed free at: http://genesdev.cshlp.org/content/19/17/1968.full.html\#ref-list-1

\section{License}

Email Alerting

Receive free email alerts when new articles cite this article - sign up in the box at the top Service 